\title{
Microvascular Cricoid Cartilage Reconstruction With the Thoracodorsal Artery Scapular Tip Autogenous Transplant
}

Eric J. P. Chanowski, MD, MPH; Marc J. Haxer, MA, CCC; Douglas B. Chepeha, MD, MSPH

Conservation laryngeal surgery has been limited by difficulties with partial resection of the cricoid. Numerous options have been suggested that include temporoparietal flaps, free cartilage grafts, radial forearm free tissue transfers, and tracheal autotransplantation with vascular carriers. The authors present a one-stage procedure for the reconstruction of the cricoid cartilage based on the thoracodorsal artery scapular tip (Tdast) autogenous transplant that uses the curved tip of the scapula and does not create a secondary tracheal defect. Because the Tdast is a vascularized graft it may withstand radiation treatment.

Key Words: Thoracodorsal artery scapular tip transplant, scapular angle, scapular tip, cricoid cartilage, cricoid reconstruction.

Laryngoscope, 122:282-285, 2012

\section{INTRODUCTION}

Conservation laryngeal surgery that involves resection of part of the cricoid cartilage has few solutions because of the need to maintain a stable airway, to prevent aspiration during deglutition, and to maximize vocal function. In addition to restoring the cartilaginous framework of the larynx, promotion of primary healing and prevention of granulation and subsequent stenosis are important goals of reconstruction. To accomplish these goals, numerous options have been suggested that include temporoparietal flaps, free cartilage grafts, radial forearm free tissue transfers, and tracheal autotransplantation with vascular carriers. ${ }^{1-6}$ Delaere et al. have pioneered the reconstruction of the cricoid with a rigorous scientific methodology that has transformed the approach to cricoid resection and reconstruction. They have developed a procedure to restore the cartilaginous framework of the larynx in two stages. In the first stage, an extended hemilaryngectomy completely removes the

From the Department of Otolaryngology-Head and Neck Surgery (E.J.P.C., M.J.H., D.B.C.), and Department of Speech and Language Pathology (м.J.H.), University of Michigan Health System, Ann Arbor, Michigan, U.S.A.

Editor's Note: This Manuscript was accepted for publication September 16, 2011.

The authors have no funding, financial relationships, or conflicts of interest to disclose.

Send correspondence to Douglas B. Chepeha, MD, Associate Professor, Director of Microvascular Reconstructive Surgery, Department of Otolaryngology-Head and Neck Surgery, University of Michigan Health System, 1904 Taubman Center, 1500 E. Medical Center Drive, Ann Arbor, MI 48109-0312. E-mail: dchepeha@umich.edu

DOI: 10.1002/lary.22386 tumor, and a radial forearm autogenous transplant comprised of a skin paddle and a fascial paddle are used to restore the laryngeal defect and wrap the upper portion of the cervical trachea. In the second stage, the skin paddle from the larynx is removed except for a deepithelialized portion posteriorly, and the composite tracheal/forearm custom flap is transposed to reconstruct the larynx. ${ }^{1}$ Herein, the authors present a onestage procedure for the reconstruction of the cricoid cartilage based on the thoracodorsal artery scapular tip autogenous transplant (Tdast) that uses the curved cartilaginous tip of the scapula and does not create a secondary tracheal defect.

\section{CASE REPORT}

A 57-year-old woman presented for consultation after an incidental finding of a grade 1 chondrosarcoma of the right subglottis (Fig. 1). She was not stridorous or dyspneic on examination, and flexible nasal laryngoscopy showed a right subglottic lesion that measured $1.1 \mathrm{~cm}$ in the anteroposterior direction and involved the right cricoarytenoid joint.

A laryngofissure approach was used for the right cricoid resection. Approximately $60 \%$ of the cricoid was removed with the resection extending from $4 \mathrm{~mm}$ left of the midline anteriorly to the midline posteriorly. The right posterior cricoarytenoid (PCA) muscle was sharply dissected from the cricoid leaving a small cuff of muscle. The posterior branch of the recurrent laryngeal nerve was preserved. Next, the inferior cornu of the right thyroid cartilage was removed with the right cricoid, and 


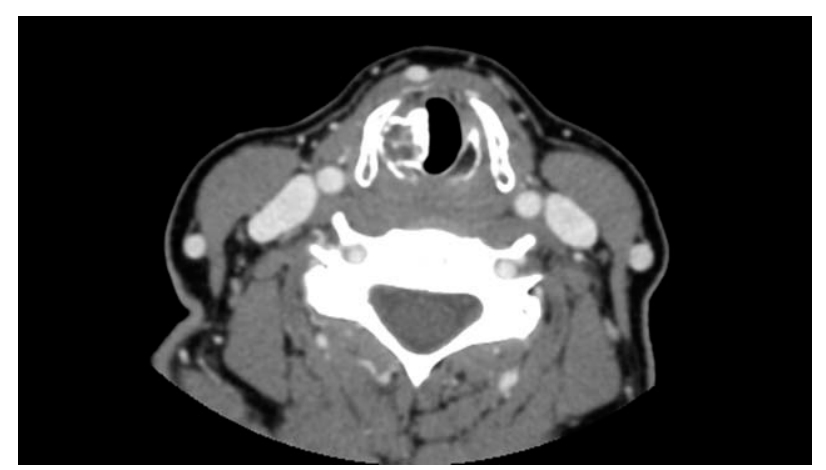

Fig. 1. Preoperative computed tomography scan of a 57-year-old woman with a grade I chondrosarcoma of the right cricoid cartilage. This lesion measured $1.1 \mathrm{~cm}$ in the anteroposterior direction and involved the right cricoarytenoid joint. A laryngofissure approach was used to resect this lesion, and a thoracodorsal artery scapular tip autogenous transplant was used to reconstruct the cricoid cartilage.

this resulted in resection of the right cricothyroid joint. The synovium between the right arytenoid and the cricoid was incised, and the right arytenoid was preserved. All margins were negative.

The Tdast was harvested in a previously reported fashion $^{7}$ to provide a $2.5-\mathrm{cm}$ piece of scapular tip bone. Next, the curved scapular tip bone was customized for inset into the cricoid defect. A cutting bur was used to fashion the scapular tip bone into a $\mathrm{C}$ shape by preserving the edge of the scapular tip and removing the central portion of the bone. Enough bone was removed so that the internal diameter of the reconstructed cricoid was larger than the internal diameter of the resected cricoid. The internal part on the $\mathrm{C}$-shaped bone would face into the airway, so that a slip of vascularized subscapularis and infraspinatus muscle could be sutured over the cut surface of bone to provide soft tissue coverage and a vascularized substrate for the buccal mucosal graft. Insetting the scapular tip transplant was done in steps. First, four 2.0 polydioxanone sutures (PDSs) were placed between the thyroid cartilage and the trachea ipsilateral to the defect. They were not tied at this time. The technique used was similar to the technique used for primary tracheal anastomosis after tracheal resection. Next, the buccal mucosal graft was placed just lateral to the intraluminal pass of the 2.0 PDSs. Using a taper needle, the $1 \times 3-\mathrm{cm}$ buccal mucosal graft was sutured into the defect with interrupted 4.0 Monocryl sutures in a circumferential fashion to provide intraluminal epithelial coverage. Next, the scapular tip was fitted into the defect. Great care was taken to ensure that the bone adjacent to the intraluminal portion of the airway was covered with muscle as described above. Once in position, the scapular tip was sutured to the remaining cricoid with interrupted 2.0 PDSs using a taper needle. Then, the innervated right PCA was put on gentle tension and draped over the posterior aspect of the scapular tip and tacked to the underlying scapular tip muscle with 4.0 Monocryl. The muscle of the scapular tip was relatively bulky; therefore the position of the PCA was approximate. Next, preparation was made to tie the 2.0 PDSs that were placed at the beginning of the inset. Care was taken not to strangulate the pedicle of the scapular tip. To avoid strangulation, the suture was passed between the bone and the muscle of the transplant on its lateral surface. All four 2.0 PDSs were placed on tension simultaneously, and the defect between the trachea and the reconstructed cricoid was closed. Because the 2.0 PDSs were intraluminal, they bolstered the buccal mucosal graft to the vascularized muscle of the scapular tip (Fig. 2). There were no early or late complications of surgery. The patient was taken to the operating room three times during her first postoperative month to laser any granulation tissue. The patient was managed postoperatively in a similar fashion as a pediatric patient undergoing subglottic reconstruction. Ciprofloxacin ophthalmic drops were placed through her tracheostomy tube three times per day, and she was placed on antireflux agents. She underwent four microlaryngoscopies postoperatively and received topical mitomycin $\mathrm{C}$ on two occasions.

\section{RESULTS}

Outpatient assessment by speech and language pathology 3 weeks postoperatively estimated that the patient was able to speak with $90 \%$ intelligibility to an educated listener. Her feeding tube was removed 1 month postoperatively, and she was able to maintain oral nutrition. She was decannulated 11 weeks after treatment, and she returned to full-time work 3 months postoperatively, where she uses the phone for several hours per day. Thirteen months postoperatively the patient has remained free of disease and she reported no functional issues in any of the categories of the Head and Neck Speech and Swallowing Assessment (Fig. 3). ${ }^{8}$ Her overall score was $92.5 / 100$ on the University of Michigan Voice Related Quality-of- Life Assessment. ${ }^{9}$ On this 10-item instrument, less than perfect scores were reported for two items: being heard in noisy situations

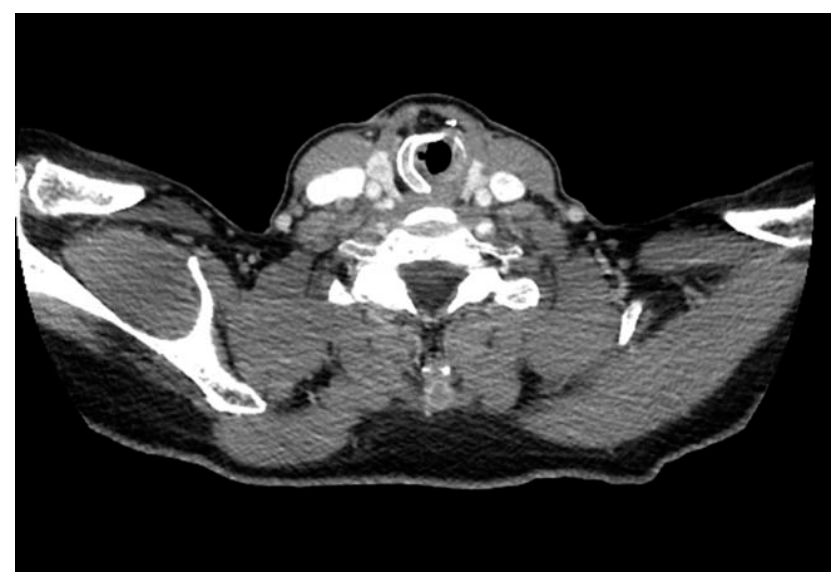

Fig. 2. A 9-month postoperative computed tomography scan of the patient in Figure 1. The bone of the scapula tip is shown in the anterosuperior portion of the figure, reconstructing the right $60 \%$ cricoid defect that is restoring the cartilaginous framework of the larynx. The airway is patent and the bone of the scapula tip has not filled in over time. 

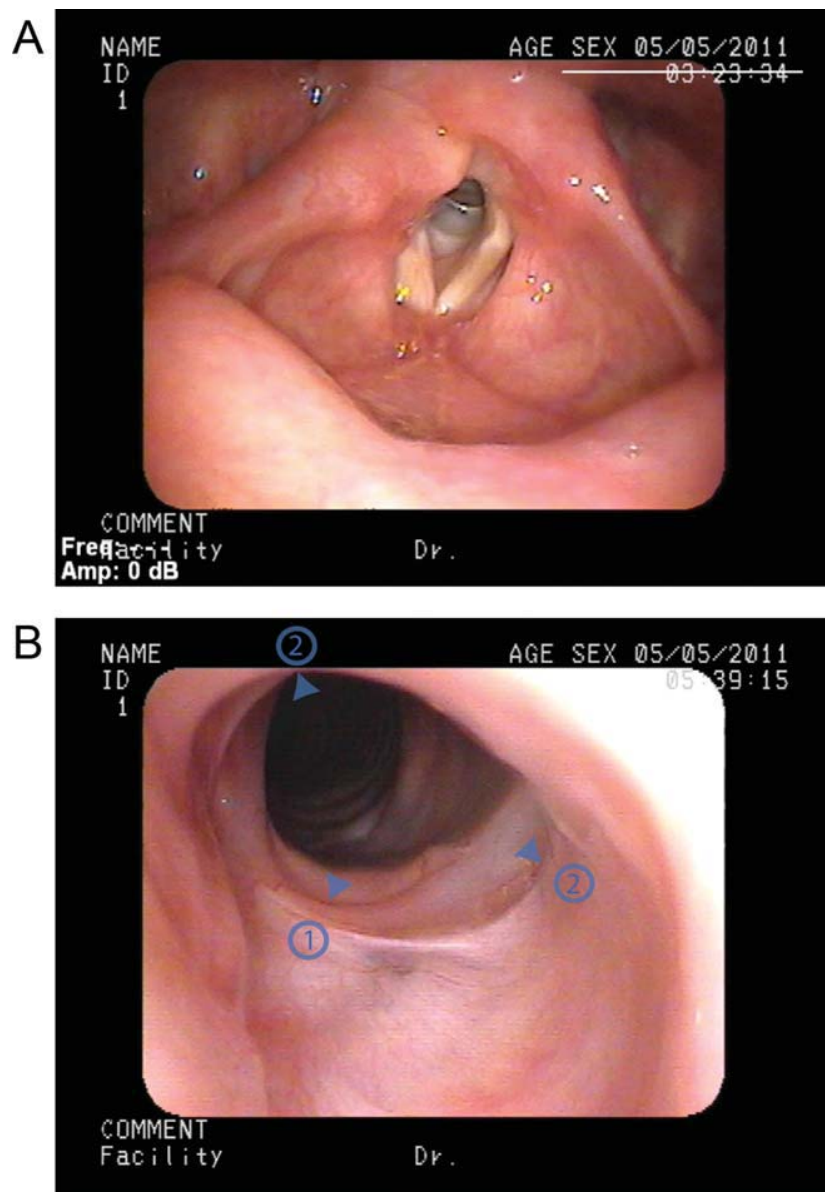

Fig. 3. (A) A 13-month postoperative endoscopic image of the glottis. The inferior aspect of the figure is the anterior surface of the larynx. The left side of the figure is the patient's right side. The patient's right cord is mobile, but the mobility is limited. The cord is shortened and not tight as on the left. She is able to abduct her right cord but has decreased strength of adduction. The scapula tip bone can be seen subglottically on the patient's right. (B) A 13-month postoperative endoscopic image of the subglottis and trachea. The inferior aspect of the figure is the anterior surface of the trachea. The left side of the figure is the patient's right side that is reconstructed with the scapular tip. The patient's airway is patent. The scapula tip bone used to reconstruct the right cricoid cartilage defect is seen on the patient's right side. The tracheoscapular junction (1) and the crico-scapular junctions (2) are shown. There is no exposed bone, and the bone of the scapula tip has not filled in during follow-up.

(raw score, 3/5) and having to repeat herself to be understood (raw score, 2/5). The patient scores less than perfectly for these items because she struggles when speaking with clients during corporate dinners in noisy environments.

\section{DISCUSSION}

Conservation laryngeal surgery has been limited by difficulties with partial resection of the cricoid. The ideal reconstructive approach for conservation laryngeal surgery would restore the cartilaginous framework of the larynx, provide an epithelial lining for the reconstructed endolarynx, promote primary healing, prevent granulation, be a single-stage procedure, yield minimal secondary defects or deficits, and be durable, even in a field that has or will undergo radiation. Other than the procedure pioneered by Delaere and his colleagues at the University Hospital in Leuven, Belgium, available techniques for cricoid reconstruction have remained limited. Reconstruction with the Tdast offers threedimensional vascularized bone that can be shaped to recreate the cartilaginous framework of the larynx, is performed in a single stage, yields minimal donor-site morbidity, has ample soft tissue from the latissimus muscle for reconstruction even in potentially radiated fields, and allows for epithelial resurfacing of the endolarynx with a buccal mucosa graft.

Previous attempts at laryngotracheal reconstruction with free tissue transfers have been reported. Gilbert and Neligan have summarized attempts at pharyngolaryngeal reconstruction with radial forearm free tissue transfers, ${ }^{2}$ and Urken et al. have reported the use of the radial forearm free tissue transfer with a free cartilage graft to reconstruct the hemicricoid. ${ }^{5}$ However, concern remains over the use of free cartilage grafts in a contaminated field that has or will undergo radiation. Gilbert and Neligan have shown excellent results with the use of the temporoparietal free flap as a vascular carrier for a cartilage graft for patients undergoing hemilaryngectomy with preservation of the cricoid after radiation failure. ${ }^{2}$ Mayot et al. reported the results of three innovative cases, in which the cricoid cartilage was reconstructed after vertical or anterior hemilaryngectomy using free vascularized bone from the lateral border of the scapula based on the circumflex scapular artery. $^{3}$ Radiation therapy had no adverse effects on the vascularized bone at follow-up intervals of 10 to 18 months, but the bone could not be contoured into a partial circle. Alternatively, the Tdast can be contoured into a partial circle without osteotomy by using a reciprocating saw or cutting bur. The bone of the scapula tip did not fill in over time after contouring and inset (Figs. 2 and 3), which can occur with other osseous donor sites such as the lateral border of the scapula and the fibula. This may be because the curved natural shape of the scapula tip was contoured along embryologic growth planes, ${ }^{10}$ which does not occur when the aforementioned osseous donor sites are used for cricoid reconstruction.

Excellent techniques have also been reported for laryngotracheal reconstruction that uses tracheal autotransplantation in single- or multistage procedures. ${ }^{1,6}$ These techniques result in secondary tracheal defects requiring one- or two-stage procedures to reconstruct. The scapula tip donor site may offer new options compared to the trachea because it is not part of the airway, and its harvest results in no secondary tracheal defect. However, tracheal autotransplantation offers the ability to transport multiple tracheal rings cephalad, which continues to make it an attractive technique for the surgical management of tracheal stenosis. Zur and Urken report excellent results using hemitracheal autografts, with the thyroid gland as a vascular carrier for subglottic stenosis, and comment on the potential use of this technique for unilateral laryngeal sarcomas. ${ }^{6}$ However, we agree with their concern in using this technique for epithelial laryngeal neoplasms because 
of the risk of occult metastases to the paratracheal lymph nodes that can be seen with epithelial tumors having significant subglottic extension. ${ }^{4,6}$

Our patient reported excellent postoperative speech and swallowing function. Her results compare favorably with those previously reported,,$^{2,3,5,6}$ and her ability to return to a profession in which she regularly uses her voice reflects her confidence in her ability to communicate and be understood.

\section{CONCLUSION}

The Tdast is an interesting new approach for reconstruction of extended hemicricoid defects after conservation laryngeal surgery. Because it is a vascularized graft, it may be a better choice for patients who have undergone or have a treatment plan that includes postoperative radiation treatment. In addition, this approach has the advantage of a one-stage technique that provides nonadjacent tissue that very specifically reduplicates the curved shape of the cricoid cartilage.

\section{BIBLIOGRAPHY}

1. Delaere P, Vander Poorten V, Vranckx J, Hierner R. Laryngeal repair after resection of advanced cancer: an optimal reconstructive protocol. Eur Arch Otorhinolaryngol 2005;262:910-916.

2. Gilbert RW, Neligan PC. Microsurgical laryngotracheal reconstruction Clin Plast Surg 2005;32:293-301, v.

3. Mayot D, Widmer S, Bichet G, Braun M, Lindas P, Perrin C. Use of a scapular free flap for reconstruction of the cricoid cartilage in pharyngolaryngeal oncology. Arch Otolaryngol Head Neck Surg 1994;120: 662-667.

4. Urken ML. Advances in head and neck reconstruction. Laryngoscope 2003; 113:1473-1476.

5. Urken ML, Blackwell K, Biller HF. Reconstruction of the laryngopharynx after hemicricoid/hemithyroid cartilage resection. Preliminary functional results. Arch Otolaryngol Head Neck Surg 1997;123:1213-1222.

6. Zur KB, Urken ML. Vascularized hemitracheal autograft for laryngotracheal reconstruction: a new surgical technique based on the thyroid gland as a vascular carrier. Laryngoscope 2003;113:1494-1498.

7. Chepeha DB, Khariwala SS, Chanowski EJ, et al. Thoracodorsal artery scapular tip autogenous transplant: vascularized bone with a long pedicle and flexible soft tissue. Arch Otolaryngol Head Neck Surg 2010;136: 958-964.

8. Chepeha DB, Teknos TN, Shargorodsky J, et al. Rectangle tongue template for reconstruction of the hemiglossectomy defect. Arch Otolaryngol Head Neck Surg 2008;134:993-998.

9. Hogikyan ND, Sethuraman G. Validation of an instrument to measure voice-related quality of life (V-RQOL). J Voice 1999;13:557-569.

10. Teot L, Souyris F, Bosse JP. Pedicle scapular apophysis transplantation in congenital limb malformations. Ann Plast Surg 1992;29:332-340. 